\title{
Objet et démarche de la tectonophysique
}

\author{
Topics and procedures in tectonophysics
}

\author{
P. VIALON \\ LGIT-IRIGM. Observatoire de Grenoble - Université Joseph-Fourier*
}

Rev. Franç. Géotech. n० 56, pp. 7-13 (juillet 1991)

\section{Résumé}

Sous le contrôle de l'observation du terrain, la tectonophysique utilise les méthodes, les techniques ou règles de la physique, de la mécanique, de la chimie... pour tenter de reconstituer des lois du comportement des roches valables dans des conditions géologiques. Ces mécanismes, bien compris, devraient permettre de prévoir le comportement de structures naturelles sous de nouvelles sollicitations introduites par l'homme et à d'autres échelles d'espace et de temps.

Cette note montre avec quelques exemples, quelle est la démarche qui permet de conduire des caractères structuraux essentiels d'un massif à des lois de valeur générale expliquant les mécanismes de déformation géologique. Le rôle majeur de la dissolution des roches et du transfert puis de la précipitation des solutions, est souligné. Ces mécanismes associés expliquent souvent le fluage lent, mais néanmoins significatif à l'échelle des temps géologiques, qui se produit dans les dix premiers kilomètres de la croûte terrestre. Ils permettent aussi de comprendre des structures dont la coexistence et la contemporanéité paraissent paradoxales voire incongrues selon les règles des classiques comportements utilisés par l'ingénieur.

\begin{abstract}
Tectonophysics, controlled by field observations, uses the methods, techniques, and laws of physics, mechanics and chemical sciences while applying them to the natural deformations of the earth crust. The aim of this paper is to illustrate how structural characteristics of rocks can be used to infer general laws determining deformation paths and mechanisms acting at a geological scale of space and time. Thus, predictive behaviours, under new human scale solicitations, can be derived for the deformed rock materials and massifs.

Under geological conditions, the upper earth crust seems to behave paradoxically at the same time as brittle and ductile materials. This can be explained by the fluid control of the deformation, acting by dissolution, then transfer and redeposition of solutions. This flowing of rocks, at a very slow strain rate which is, nevertheless, significant at a geological scale, must be superimposed to the classical, and simpler, pressure and temperature dependent behaviours.
\end{abstract}

* BP 53X, 38041 Grenoble Cedex. 
Les sciences géologiques possèdent, depuis quelques années, une nouvelle orientation parmi leurs multiples facettes: la tectonophysique. Son but est de mieux cerner, dans le cadre d'une discipline qui se veut originale, le comportement des roches dans leurs conditions géologiques de déformation.

Il semble que l'origine du mot, et des concepts qu'il recouvre, soit à rechercher, bien avant la parution du premier titre de la revue spécialisée bien connue qui porte ce nom, dans l'ouvrage de JAEGER et COOK (1) "Fundamentals of rock mechanics ». Au moins dans les éditions qui suivirent la première (1969), sans en modifier l'essentiel, la tectonophysique est définie comme une branche de la mécanique des roches dédiée à l'étude, dans la terre, des relations entre forces et déformations. Bien que par la suite $(2,3$ et 4) le mot ait été utilisé dans une acception plus restreinte, il est clair que désormais il sert à désigner l'ensemble des études qui tentent, avec les moyens de la physique, de la mécanique.... de comprendre comment les roches et les massifs ont enregistré l'histoire de leurs déformations (structures, mécanismes, conditions, comportements, etc.) replacées dans leur échelle géologique d'espace et de temps.

C'est en ce sens que tectonophysique et mécanique des roches se différencient. Moyens, méthodes, parfois langage, peuvent être voisins, mais le but essentiel est moins, pour l'ingénieur, de remonter (comme tente de le faire le tectonophysicien) dans la mémoire du matériau, que d'en définir le comportement et la résistance dans sa configuration actuelle (5).

Il est bien admis que l'organisation présente du matériau rocheux, à l'échelle de l'échantillon comme à celle du massif, résulte d'une suite de déformations plus ou moins compliquées. Les chaînes de montagnes sont l'expression majuscule et en effet exemplaire, des déformations naturelles de la croûte terrestre. Comme l'architecture des chaînes de montagnes s'identifie souvent à la "tectonique ", il y a fréquemment un glissement sémantique : les objectifs de la tectonophysique se situeraient dans le domaine de la tectonique, c'est-à-dire en pays montagneux, exclusivement.

Certes les montagnes sont des lieux favorables pour étudier les déformations de la terre. La topographie, l'excellence des affleurements rocheux, permettent d'y avoir aisément accès à différents aspects et conditions de déformations de la croûte terrestre, dans son épaisseur. Comme, de plus, l'érosion peut atteindre les racines exhumées d'une vieille chaîne, (qui dans une région alors pénéplanée n'a plus guère droit au qualificatif géographique de montagnes) les résultats de la déformation des roches à grande profondeur sont donc également accessibles. Et ce sont même les déformations de la croûte océanique qui peuvent être étudiées dans les chaînes de montagnes puisque l'accrétion continentale y cicatrise les océans perdus (6).

Mais ce large éventail de matériaux, conditions, mécanismes... auquel les montagnes donnent accès pour étudier les déformations terrestres en situation, ne doit pas cacher qu'il est d'autres régions où les roches sont également déformées, peut être à degré moindre et dans d'autres conditions, mais qui n'en sont pas moins du domaine d'étude de la tectonophysique.
Les roches des régions de la terre qui ne sont pas, n'ont jamais été et ne sont sans doute pas destinées à être jamais dans une chaîne de montagnes, sont en effet toujours plus ou moins déformées. En fait toute roche, dès que formée, ou en cours de formation, se structure, de l'échelle intragranulaire à celle du massif, et ainsi se déforme (7). Un sédiment expulse l'eau dont il est imbibé, et au cours de son dépôt commence à se déformer par tassement. La consolidation précède d'éventuelles nouvelles sollicitations qui introduiraient la superposition de nouvelles structures. De même, un magma fondu commence à s'organiser en s'écoulant tout en refroidissant et cristallisant avant d'être éventuellement déformé à nouveau sous d'autres conditions. Une roche devient donc nécessairement, et surtout si elle appartient à un domaine orogénique, un composite à la mémoire chargée de déformations successives enregistrées sous diverses conditions depuis sa formation.

Dans ce matériau jamais simple, peuvent cependant se rencontrer des simplifications permettant d'analy. ser des comportements caractéristiques des déformations géologiques. La qualité de la roche, les conditions, l'intensité de la déformation... peuvent rendre homogène la structure finale au point que tous ses caractères appartiennent et décrivent le seul comportement enregistré dans les conditions les plus extrêmes. Mais la déformation peut être au contraire très hétérogène et dans ses différentes localisations se lisent alors les caractères de différents incréments, représentatifs du comportement d'une époque dans l'histoire de la roche. Le problème sera alors de savoir relier chronologiquement ces différents caractères, ou d'y établir une discrimination s'ils se trouvent associés en un mélange. En ce cas la question est de distinguer entre elles les différentes contributions à la déformation finie dans ce stock global de l'enregistrement des réactions, sous différentes conditions, d'un matériau évolutif à mémoire.

\section{DES COEXISTENCES STRUCTURALES INCONGRUES}

II n'est pas possible ici de passer en revue tous les thèmes d'étude de la tectonophysique. Aussi, dans le but d'illustrer la démarche du tectonophysicien, quelques cas particuliers choisis sont analysés. Il s'agit d'exemples, très courants, de déformations localisées sur des discontinuités aux caractères très tranchés. Les structures paraissent résulter d'un comportement du matériau rocheux qui serait à la fois, et en même temps ductile et cassant. Pour qualifier mécaniquement plus précisément ces comportements associés, réputés appartenir à des conditions de déformation assez distinctes, il convient d'envisager des phénomènes qui ne sont pas uniquement dépendants de la pression et de la température. On montre comment il est possible de prendre en compte la dimension géologique du problème en centrant l'attention, autre choix parmi d'autres cas d'espèces, sur le rôle des fluides (8). 


\subsection{Les systèmes de fractures " en échelons"}

Le déplacement relatif de deux parties d'un massif, sur quelques centimètres ou quelques centaines de mètres, voire plus, peut se produire sur une limite commune unique dite discontinuité, plan de rupture ou faille. C'est sur ce plan qu'a lieu le glissementdéplacement rigide entre les deux compartiments découpés dans le massif.

Mais pour des objets de mêmes dimensions le plan idéal de glissement peut être remplacé par une zone diffuse plus ou moins épaisse (9) (quelques centimètres à quelques mètres) où s'alignent de façon non quelconque de petites fractures élémentaires dites « en échelons $\%$. La figure 1 schématise de telles zones. Cette organisation correspond à la déformation par cisaillement, due au frottement, du bord des deux compartiments déplacés. La zone de cisaillement constitue ainsi "l'échelle " sur laquelle se disposent les échelons représentés par les fractures élémentaires. Sur la figure 1 les flèches extérieures aux bandes cisaillées-fracturées indiquent le déplacement général relatif entre les deux compartiments du massif. En A on voit des zones jalonnées par une seule famille de fractures, plus ou moins tordues par le déplacement et jointives : "faille tressée ». Toute la zone où se concentrent le déplacement et la déformation est alors découpée en petits blocs dont les glissements et rotations les uns par rapport aux autres confèrent à la zone une " plasticité en grand " et permettent le déplacement d'ensemble (10).

Le croquis A1 montre les blocs lenticulaires découpés par les seules fractures $\mathrm{R}$ (voir ci-après), devenues sigmoïdes en raison des déplacements. Le croquis A2 montre des échelons de fractures T (voir plus loin).

En changeant l'échelle de l'observation de ces structures rapidement interprétées, l'alignement des peti-

A1
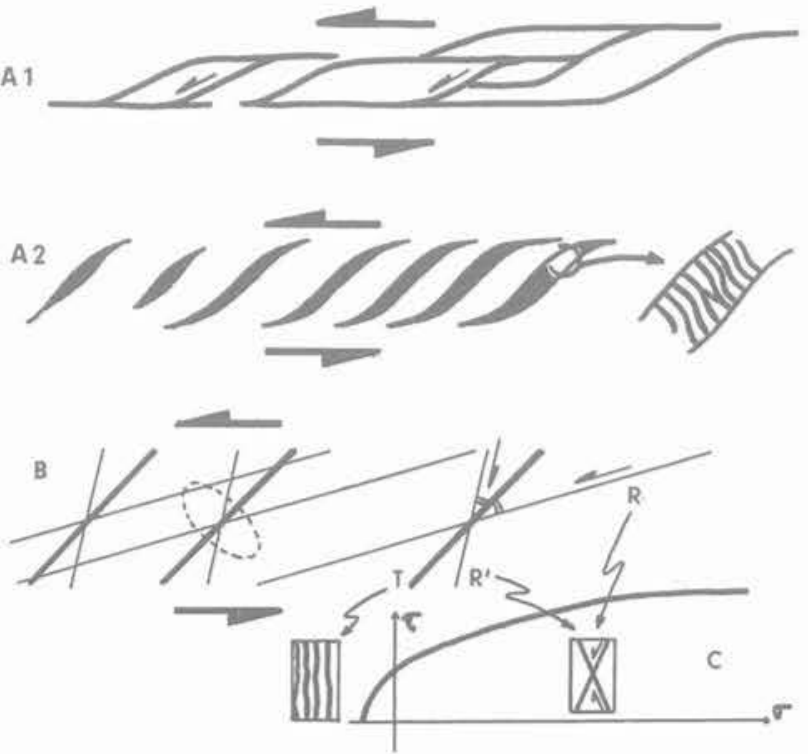

Fig. 1. - Zones de cisaillement avec fractures "en échelons " Fig. 1. - Shear zones with "en échelons " arrays of veins or fractures. tes ruptures peut également paraître correspondre à une fracturation précoce (11). L'ensemble de la zone délimitant le futur déplacement tangentiel entre deux compartiments du massif serait fragilisé par des fractures en mode I. L'évolution du système, en fonction des conditions aux limites imposées, entrainerait ultérieurement l'apparition de la discontinuité macroscopique en mode II : plan majeur rassemblant les petites fractures précoces et sur lequel se produit le glissement rigide.

Un autre type d'analyse peut aussi prendre en compte la qualité structurale des différentes fractures associées. Les fractures $T$, dites d'extension, sont souvent ouvertes ou comblées par des cristallisations syncinématiques (cristaux fibreux sigmoïdes orientés par l'ouverture et la torsion progressives de la fissure hôte : voir fig. 1, A2, le détail cerclé) (12). Elles se situent dans le plan bissecteur des fractures conjuguées $\mathrm{R}$ et $\mathrm{R}^{\prime}$ et sur lesquelles de petits déplacements provoquent des stries de frottement souvent prolongées par des fibres cristallines de même orientation $(7,9)$. Les glissements comme la croissance suncinématique des fibres et l'organisation géométrique du système de fractures sont harmonieusement cohérents et paraissent le justifier.

La figure $1 \mathrm{~B}$ indique ce système complet de fractures échelonnées d'une zone de cisaillement. $\mathrm{T}$, parallèle à la direction d'aplatissement de la zone (une ellipse, déformée globale d'un cercle initial, situe les directions principales avant la torsion qu'entraînerait un plus fort déplacement général) est bissectrice du réseau de fractures conjuguées $\mathrm{R}$ et $\mathrm{R}$ ' (dit de Riedel) où les déplacements symétriques sont indiqués par des flèches.

Toutes ces analyses semblent avoir une parfaite logique géométrique et cinématique liée au déplacement des compartiments découpés dans le massif. Pourtant plusieurs incompatibilités, apparentes, méritent d'être soulignées dans cette association de structures:

- la succession : petites fractures en mode I suivies d'une plus grande en mode II, ne correspond pas à la coexistence des $T+R$ et $\mathrm{R}^{\prime}$ dans la zone localisant la déformation dans le futur plan de déplacement ;

- si les fractures $\mathrm{T}$ sont du domaine fragile, puisqu'admises parallèles à la contrainte principale majeure du système, avec des dispositifs de détail qui indiquent une éventuelle assistance à la fracturation par la pression des fluides, elles correspondent donc à une création sous faible contrainte moyenne, alors que..

- le réseau $R+R$, qui coexiste avec les fractures $\mathrm{T}$ contemporaines appartient à un système conjugué de ruptures de Mohr-Coulomb, de type pseudoplastique, réputé apparaître au contraire sous assez forte contrainte moyenne (voir fig. $1 \mathrm{C}$ qui situe sur un schéma de Mohr les différentes familles de fractures avec une courbe de rupture idéalisée et l'aspect des éprouvettes correspondantes) ;

- l'instantanéité de la rupture de l'une ou l'autre de ces familles de fractures ne paraît pas vérifiée par les remplissages de cristaux dont la croissance est guidée par les déplacements, et la cinétique de cristallisation semble devoir intervenir sur la vitesse de déformation et de déplacement de l'ensemble. 
Au total, le comportement géologique qui explique la déformation d'une zone de fractures en échelons, paraît bien dépendre de la résistance à la rupture du matériau initial, avec assistance ou non par la pression des fluides. Mais la coexistence et l'évolution simultanée des structures associées, impliquent aussi que la déformation soit commandée par les phénomènes qui permettent la naissance des cristaux syncinématiques. Différents mécanismes interviennent donc nécessairement et simultanément pour que le déplacement se produise selon la zone où s'alignent les échelons. Le plus lent d'entre eux déterminera la vitesse de déformation de tout le système (13). Il s'agit :

- de la rupture et de son évolution (vitesse de propagation, d'ouverture et de glissement) :

- de la mise en solution de minéraux qui ensuite précipitent dans les vides ménagés par les fractures; - du transfert des solutions depuis les zones de dissolution vers les zones de dépôt :

- de la vitesse de cristallisation.

Ainsi le comportement géologique d'un tel système doit-il être décrit dans sa dépendance de la pression solide et fluide, ce qui est banal mécaniquement, mais aussi en prenant en compte des facteurs qui dépendent beaucoup plus du temps et de l'activité chimique des phases en présence et sans l'intervention desquels la structure observée ne serait pas activée, ni cohérente ou expliquée par les lois mécaniques traditionnelles.

\subsection{La schistosité associée à des fractures d'extension}

La schistosité des roches sédimentaires déformées (surtout argileuses et marneuses), est réputée caractériser et par excellence, le comportement ductile des roches dans les conditions naturelles de leur déformation. Le matériau doit être situé sous forte contrainte moyenne et fort déviateur ou/et avoir une composition favorable. Dans les roches argileuses on retient surtout la présence d'eau, qui si elle n'est pas expulsée au cours de la déformation, peut permettre une certaine réversibilité de celle-ci ou des superpositions de schistosités $(13,14)$.

La structure schisteuse est due à une orientation préférentielle des grains de la roche (surtout efficace pour les minéraux anisotropes : phyllosilicates divers et particulièrement argiles). Ils se disposent selon un plan d'aplatissement, évidemment perpendiculaire à la direction de contrainte principale majeure du moment. $\mathrm{Ce}$ plan d'anisotropie structurale et mécanique de la roche peut être obtenu selon différents mécanismes. On négligera ici l'orientation préférentielle acquise par glissements intracristallins activé par la température. Par contre les comportements commandés par la teneur en eau retiendront l'attention (8).

Deux processus fondamentaux sont réputés pouvoir créer la schistosité dans les roches en présence de fluides $(8,13)$ :

- la réorientation par rotation des particules; ce mécanisme s'accompagne dans l'assemblage de plus en plus dense des grains, d'une perte en eau, d'un tassement et d'un durcissement du matériau ;

- la dissolution-cristallisation (pressure-solution) (15) qui implique un changement de forme des grains; dissouts, et donc raccourcis selon la direction de plus forte compression, les grains s'allongent corrélativement sur les faces soumises à de moindres pressions, qu'il s'y produise ou non un dépôt. Des changements de composition chimique et de volume accompagnent aussi le plus souvent la dissolution-cristallisation qui correspond à des transferts en solution (16).

Dans beaucoup de cas cette déformation ductile est associée à une fracturation perpendiculaire au plan de schistosité. Cette fracture permet la cristallisation de minéraux dont la croissance se fait en fibres au fur et à mesure de son ouverture (12). Comme dans l'exemple précédent cette fissure est dénommée $\mathrm{T}$ pour souligner ses caractères d'extension et sa position transversale au plan d'aplatissement. Mais comme précédemment aussi, l'analyse purement mécanique de cette déformation qui ne tiendrait compte que de sa dépendance des pressions solide et fluide et/ou (ici très accessoirement) de la température, est insuffisante. Pour rendre compte de cette coexistence apparemment paradoxale de figures de déformation en même temps ductile et fragile, la tectonophysique fait intervenir l'activité chimique des phases en présence et les vitesses de transfert.

La figure 2 illustre ces cas : la schistosité $\mathrm{S} 1$, soulignée par un litage tectonique qui correspond à l'accumulation de produits insolubles (argiles) concentrés dans le plan d'aplatissement-dissolution, est plan axial de plis modérés des strates (SO) de marnes du Jurassique dauphinois. Les carbonates dissouts sont transférés dans des fissures d'extension $\mathrm{T}$ perpendiculaires. Remarquer que les fissures $T$ recoupent ou sont recoupées par S1, ce qui indique la contemporanéité de ces deux plans structuraux.

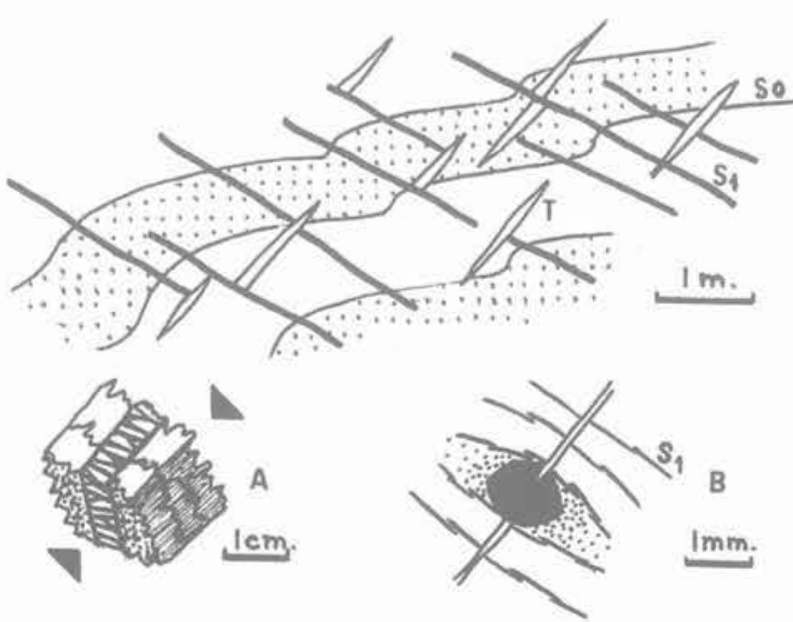

Fig. 2. - Schistosité de dissolution-cristallisation associée à des fractures d'extension perpendiculaires (d'après photographie: galerie EDF sous le massif de Bramefarine entre Allevard et Le Cheylas-(sère). Fig. 2. - Pressure-solution cleavage associated to perpendicular extension veins (from a photograph in a EDF tunnel near Allevard-Isère). 
Dans les schémas $A$ et $B$ annexés et orientés de manière identique, sont indiquées les analogies (noter les différences d'échelle) avec :

A : la déformation par stylolitisation des calcaires avec dépôt des éléments dissouts sur le joint stylolitique dans la fissure perpendiculaire;

B : la déformation de marnes gréseuses autour d'un corps dur (cristal de pyrite). Les argiles insolubles se concentrent en fins litages tectoniques S1 moulées sur le corps dur alors que les produits mis en solution se concentrent dans les zones protégées proches du cristal de pyrite ( ombres de pression " en pointillé sur le croquis) et dans la fissure perpendiculaire au plan d'aplatissement.

\subsection{Le plan de schistosité comme fracture d'extension}

Si l'association croisée d'un plan de schistositéaplatissement et d'une fracture d'extension pose le même problème de l'activité chimique des fluides (17) et de leur transfert que les systèmes de stylolitisation des calcaires (voir fig. 2) (18), la question paraît plus complexe si le plan d'aplatissement lui-même devient le siège d'une extension (19). Le réflexe habituel est de penser à la superposition de deux régimes de déformation. Dans l'incrément le plus récent, la schistosité aurait perdu sa valeur de plan principal de déformation, perpendiculaire à la direction de compression maximale.

En fait ce changement de direction ou de hiérarchie des contraintes appliquées, n'est pas nécessaire. Pour analyser le comportement géologique d'un massif où le plan de schistosité est localement devenu une fracture ouverte emplie de cristaux syncinématiques en fibres parallèles à la direction d'aplatissement (voir fig. 3) (13), il faut toutefois tenir compte de la forte

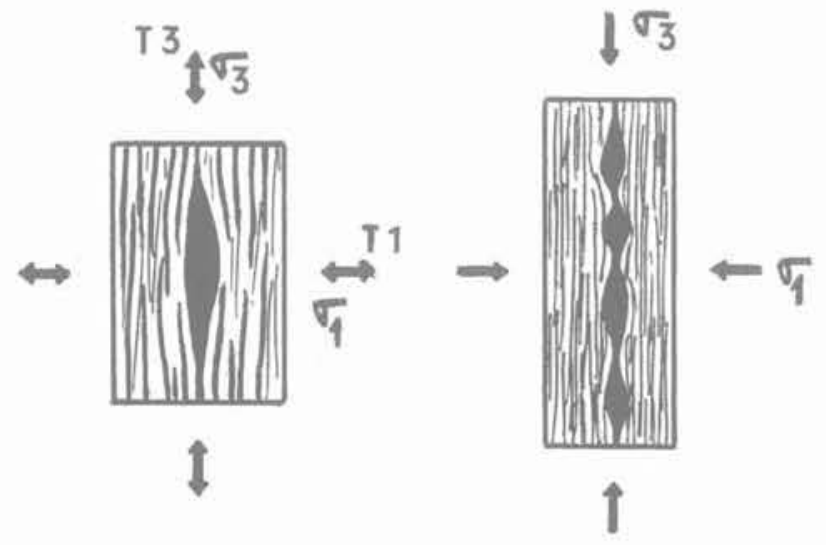

Fig. 3. - Plan de schistosité S1, ouvert et colmaté par des cristallisations. Le plan à remplissage cristallin est ensuite boudiné en raison de l'aplatissement persistant selon

$S 1 . T=$ résistance à la traction. $T 3=30 \mathrm{~T} 1$.

Si Pf $>\sigma 1+T 1$, il y a ouverture de $S 1$

à condition que $(\sigma 3+T 3)>(\sigma 1+T 1)$.

Fig. 3. - S1 cleavage plane first opened then sealed. Boudinage occurs after in consequence of the persistent

flattening in the S1 plane. $T=$ tensile stress. The opening of $S 1$ appears for the conditions given above. anisotropie mécanique acquise par la roche pendant la schistogenèse. De même, pendant celle-ci, l'importante libération de solutions corrélative à la réalisation de l'assemblage de plus en plus serré des grains, va nécessairement et au moins transitoirement, entraîner une surpression fluide dans le matériau (8). Le fluide disponible aura le double rôle d'aider la fracturation selon la direction qui offre la moindre résistance et de permettre la précipitation des éléments dissouts dans le vide créé par la fracture.

Dans les matériaux schisteux, il est banal de mesurer une résistance à la traction 30 fois plus élevée parallèlement au plan d'anisotropie que perpendiculairement $(5,20)$. Si la pression des fluides libérés par la schistogenèse dépasse la somme de la contrainte de compression perpendiculaire au plan d'aplatissement et de la résistance à la traction dans cette direction, alors il sera énergétiquement plus rentable d'ouvrir le plan d'anisotropie qui se crée que d'obtenir une nouvelle rupture perpendiculaire. $\mathrm{Ce}$ cas se produit évidemment si parallèlement à la schistosité, la somme de la contrainte de compression et de la résistance à la traction (dont on a souligné l'énorme différence avec celle qui est mesurée perpendiculairement), est plus élevée que la surpression momentanée des fluides.

La chute de pression consécutive à la rupture = ouverture du plan de schistosité, entraîne la précipitation dans le vide créé des éléments en solution et la persistance apparemment paradoxale de l'aplatissement selon ce même plan. La poursuite de ce régime se traduit par une déformation en boudinage du remplissage de la fissure parallèle à la schistosité $(19,13)$.

\section{DE L'IMPORTANCE DU RÔLE DES FLUIDES DANS LA DÉFORMATION}

Il est sans doute bien inutile de rappeler l'importance des fluides dans la déformation. Par leur pression et ses variations ils jouent un rôle toujours mieux démontré dans le comportement mécanique des sols et des roches. Mais si cette importance « géotechnique » n'est plus à souligner, il est néanmoins clair qu'une partie de leur rôle est négligée : celle qui concerne le transfert de matière en solution. C'est peutêtre pourtant ce rôle qui est le plus important dans les déformations géologiques, au moins dans les dix à quinze premiers kilomètres de la croûte terrestre.

\subsection{La dissolution-percolation}

Ce cas, le plus simple, choisi en schéma exemplaire, a un fort intérêt pratique en particulier en recherche et exploitation minières. Tout massif contient des fluides (17) qui transitent plus ou moins rapidement en fonction de la nature éventuellement hétérogène des terrains, vers les zones plus nettement perméables (grès, sables, fissures...) qui sont des drains naturels. Dans ce transit, surtout s'il est lent, les fluides, essentiellement $\mathrm{H}_{2} \mathrm{O}$, peuvent dissoudre et entraîner avec eux certains éléments constitutifs du massif et particulièrement solubles. Un cas d'espèce banal est celui 
de sels métalliques par exemple, très dispersés et à très faible teneur dans un matériel peu perméable. Le fluide qui percole très lentement se charge en ces métaux qui ultérieurement vont se déposer à plus forte concentration dans les drains plus perméables où se déversent les solutions. C'est schématiser le phénomène très courant du drainage de minerais vers les filons d'un massif par ailleurs globalement faiblement minéralisé.

Cette concentration minérale filonienne n'entraîne apparemment pas de conséquences sur les déformations du massif. Ou du moins celles-ci ne sont elles généralement pas considérées. Mais s'il s'agit d'une association d'argilites, qui se compactent, avec des grès, qui jouent le rôle de drains-concentrateurs de la minéralisation auparavant dispersée dans les argilites, il peut en être autrement. L'enrichissement des grès en éléments transférables par percolation des solutions expulsées par les argilites se complète en effet d'une compaction de ces dernières.

\subsection{Les modèles de fluage en présence de fluides}

La prise en compte des fluides et des transferts de matière que ces modèles permettent, est très clairement, dans le domaine des études en tectonophysique de ces dernières années, une avancée et l'un des résultats des plus marquants.

Pour n'en résumer que l'esprit (voir détails in J.P. GRATIER (13) et la bibliographie associée) (15, 17, etc.), il faut souligner que l'écoulement ductile d'une roche en présence de fluides implique l'intervention de trois mécanismes successifs : dissolution, transfert, cristallisation ou dépôt. C'est le plus lent de ces trois phénomènes qui déterminera la vitesse du fluage.

A partir de là, J.P. GRATIER construit plusieurs modèles selon l'importance accordée à l'un ou l'autre des mécanismes en jeu. La vitesse de fluage peut être limitée par la vitesse de diffusion de matière aux limites des grains dans un fluide immobile; mais le caractère limitant peut être la vitesse de diffusion dans le solide ou la vitesse d'infiltration (percolation) du fluide chargé en produit dissout ; ce peut être enfin la cinétique des réactions de dissolution ou de cristallisation qui est la vitesse limite.

Dans tous les cas on aboutit à l'idée d'un comportement du matériau rocheux dans les conditions géologiques, assimilables à un très lent fluage visqueux (vitesses de déformation de l'ordre de $10^{-10}$ à $10^{-15} \mathrm{sec}^{-1}$, qui sont néanmoins significatives à l'échelle des temps géologiques) (21), sous de très faibles contraintes déviatoriques. C'est un comportement très peu dépendant de la température et de la pression, mais fortement assujetti aux fluides et particulièrement à leur pouvoir solvant selon les matériaux mis en jeu.

Il convient donc d'attribuer aux fluides un rôle supplémentaire dans la déformation, en plus de leur influence mieux connue sur l'amollissement dans le domaine plastique, ou la fragmentation, le frottement et la corrosion dans le domaine cataclastique.

\section{CONCLUSIONS}

Ces exemples permettent de montrer quelle démarche utilise la tectonophysique pour comprendre les lois de comportement dans des conditions géologiques et dans l'épaisseur de la lithosphère $(22,23,24)$. Schématiquement, si l'on admet simplement un comportement cataclastique à partir de la surface, sa dépendance de la pression vérifiée expérimentalement, va entraîner un durcissement progressif des roches avec la profondeur. Mais très vite la température vient concurrencer la pression et survient alors une interférence avec un comportement plastique amollissant progressivement les roches jusqu'à l'asthénosphère où l'on admet une fusion partielle.

La figure 4 indique par les courbes en trait continu cette croissance de la résistance à la rupture avec la pression en fonction de la profondeur, puis la décroissance de la résistance au fluage avec l'augmentation de la température.

Ces comportements schématiques possèdent une seule interférence dans le cas où un matériau homogène unique compose la lithosphère : c'est le cas des domaines océaniques où les différences chimico-minéralogiques négligeables entre croûte et manteau entraînent l'existence d'un seul pic de transition fragile/ductile. Mais si des matériaux différents se superposent (cas complexes des continents), la succession de comportements plus ou moins rigides avec la profondeur devient plus compliquée: la situation des ébranlements sismiques dans les seules couches « raides "le vérifie assez bien (25).

Pourtant ces variations de comportements et particulièrement celles de la résistance des roches à la rupture ou au fluage en fonction de la profondeur, sont

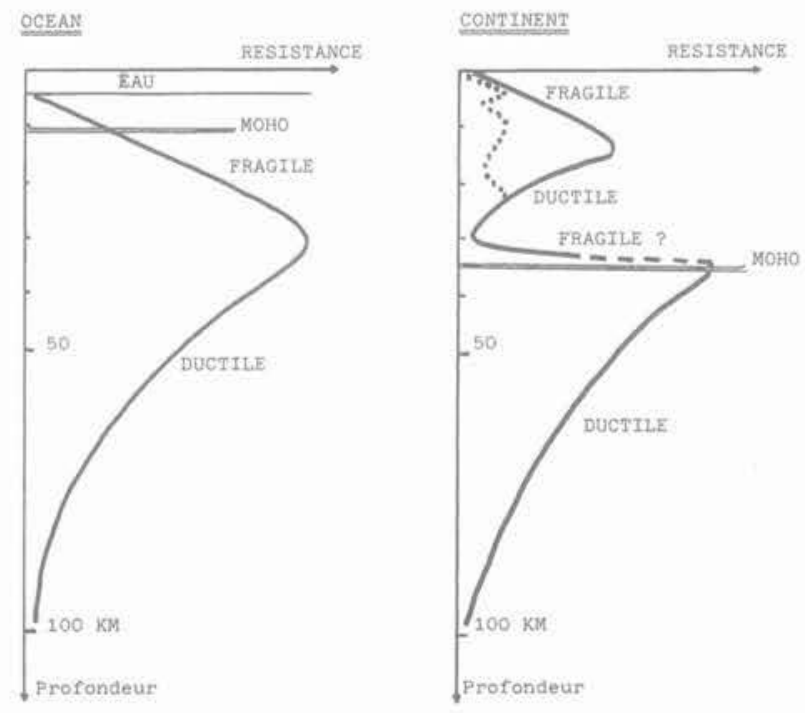

Fig. 4. - Profils de la résistance des roches dans la lithosphère océanique et continentale (en termes comparatifs : abscisses sans unités). Courbes en trait continu: résistance

à la rupture ou au fluage dépendant de la pression et de la température; courbe en pointillés: comportement en conditions géologiques.

Fig. 4. - Comparative strengths of the oceanic and continental lithosphere. Continuous lines: pressure and temperature dependent behaviours: $P$ hardening and $T$ flowing : Dash line "geological " behaviour: low strain rate, fluid transferts.. 
probablement fortement surévaluées (25). En effet dans les conditions géologiques il faut généralement admettre que les roches se sont structurées très lentement, sans efforts nécessairement rapides et importants. Par contre elles ont le plus souvent été affectées par des transferts de matière en raison de la présence d'une phase fluide.

Cette condition permet de comprendre, les exemples vus plus haut le montrent, la coexistence de structures qui devrait sans cela être expliquée par des superpositions invraisemblables. La figure 4 tente de situer les variations probables de la résistance des roches dans l'épaisseur de la croûte continentale (courbe en pointillés) dont le comportement se ferait avec transferts. Les valeurs n'en sont pas données mais la courbe doit être comparée à celle des comportements cataclastique-plastique. On remarque que les valeurs suggérées sont nettement très inférieures à celles qui sont issues des mesures en laboratoire, même obtenues sous fort confinement, haute température..., sous prétexte de simuler des conditions géologiques, qui en fait sont également autre chose.

Ces résistances « naturelles " beaucoup plus faibles sont dues essentiellement à la vitesse de déformation lente (soit des changements de taille de moins de $0,5 \mathrm{~cm} /$ an environ et par élément de $10 \mathrm{~km}$ ) avec transferts de matière grâce aux fluides. Les variations suggérées par l'allure de la courbe pointillée peuvent être dues : aux changements lithologiques, aux différences de solubilité et de porosité des roches impliquées, à la possibilité de métamorphisme et de libération corrélative de fluides, etc.

\section{BIBLIOGRAPHIE}

(1) JAEGER J.C., COOK N.G. (1971), Fundamentals of rocks mechanics. Sciences Paperback. Chapman and Hall Ed. London.

(2) LLIBOUTRY L. (1982), Tectonophysique et géodynamique. Masson Ed. Paris.

(3) NICOLAS A. (1989), Principes de tectonique. Masson Ed. Paris.

(4) FOUCAULT A., RAOULT J.F. (1984), Dictionnaire de géologie. Masson Ed. Paris.

(5) SIRIEYS P. (1966), Contribution à l'étude des lois de comportements des structures rocheuses. Thèse DE Université de Grenoble.

(6) AUBOUIN J. (1977), Tethys, Atlantique et Pacifique : regard tectonique. $\mathrm{CR}$ som. Soc. Géol. France, 4, pp. 170-187.

(7) VIALON P., RUHLAND M., GROLIER J. (1976), Eléments de tectonique analutique. Masson Ed. Paris.

(8) NICOLAS A., VIALON P. (1980), Les mécanismes de déformation ductile dans les roches. Livre Jubilaire 1830-1980, Mém. h.s. Soc. Géol. France, 10, pp. 127-139.

(9) GAMOND J.F. (1983), Displacement features associated with fault zones : a comparison between observed examples and experimental models. J. Str. Geol., 5 (1), pp. 33-45.

(10) ROBERT J.P., VIALON P. (1976), Déformation interne et déformation aux limites dans un assemblage de blocs découpés par un cisaille- ment. Le clivage schisteux des niveaux structuraux supérieurs. Bull. Soc. Geol. France, 7, 18 (6), pp. 1599-1604.

(11) POLLARD D.D., SEGALL P. (1987), Theoretical displacements and stresses near fractures in rock with applications to faults, joints, veins, dikes and solution surfaces. In ATKINSON B.K. Ed. : "Rock Fracture Mechanics ». pp. 277-349. Academic Press ED, London.

(12) RAMSAY J.G. (1980), The crack-seal mechanism of rocks deformation. Nature, 284, pp. 135-139.

(13) GRATIER J.P. (1984), La déformation des roches par dissolution-cristallisation: aspects naturels et expérimentaux. Thèse DE, Université de Grenoble.

(14) VIALON P. (1979), Les déformations continues discontinues des roches anisotropes. Eclog. Geol. Helveticae, 72/2, pp. 531-549.

(15) RUTTER E.H. (1983), Pressure-Solution in nature, theory and experiment. J. Geol. Soc. London, 140, pp. 725-740.

(16) GRATIER J.P. (1983), Estimation of volume change by comparative chemical analyses in heterogeneously deformed rocks (folds with mass transfer). J. of Str. Geol., 5, pp. 329-339.

(17) FYFE W.S., PRICE N.J., THOMPSON A.B. (1978), Fluids in the Earth crust. Elsevier Ed. Amsterdam.

(18) RISPOLI R. (1981), Stress fields about strike-slip faults infered from stylolites and tension gashes. Tectonophysics, 75, pp. T29-T36.

(19) GRATIER J.P., VIALON P. (1980), Deformation pattern in a heterogeneous material : folded and cleaved sedimentary cover immediatly overlying a crystalline basement (Oisans, France). Tectonophysics, 65, pp. 151-180.

(20) DAYRE M., KHALAF F. (1971), Etude du comportement des matériaux anisotropes schis. teux. Symposium franco-polonais : problèmes de la rhéologie. Nadbitka-Varsovie. pp. 105-119.

(21) PFIFFNER O.A., RAMSAY J.G. (1982), Constraints on geological strain rate: arguments from finite strain states of naturally deformed rocks. J. Geophys. Res., 87, B1, pp. 311-321.

(22) BRACE W.F., KOHLSTEDT D.L. (1980), Limits on lithospheric stress imposed by laboratory experiments. J. Geophys. Res., 85, B11, pp. 6248-6252.

(23) CARTER N.T., TSENN M.C (1987), Flow properties of continental lithosphere. Tectonophysics, 136, pp. 27-63.

(24) MOLNAR P. (1988), Continental tectonics in the aftermath of plate tectonics. Nature, 335, pp. 131-137.

(25) GRATIER J.P., GAMOND J.F. (1990), Transition between seismic and aseismic deformation in the upper crust. In KNIPPE R.J., RUTTER E.H., Eds : deformations mechanisms, Rheology and tectonics. Geol. Soc. London, Spec. Public., n54, pp. 461-473. 